\title{
Modelling of radio emission in the SLAC T-510 experiment using microscopic Geant4 simulations
}

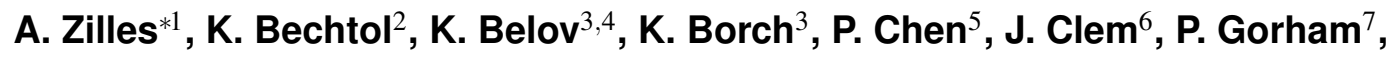 \\ C. Hast ${ }^{8}$, T. Huege ${ }^{9}$, R. Hyneman ${ }^{10}$, K. Jobe ${ }^{8}$, K. Kuwatani ${ }^{3}$, J. Lam ${ }^{3}$, T. Liu ${ }^{5}$, \\ K. Mulrey ${ }^{6}$, J. Nam ${ }^{5}$, C. Naudet ${ }^{4}$, R. Nichol ${ }^{11}$, B.F. Rauch ${ }^{12}$, A. Romero-Wolf ${ }^{4}$, \\ B. Rotter ${ }^{7}$, D. Saltzberg ${ }^{3}$, H. Schoorlemmer ${ }^{7}$, D. Seckel ${ }^{6}$, B. Strutt ${ }^{11}$, A. Vieregg ${ }^{2}$, \\ C. Williams ${ }^{13}$, and S.A. Wissel ${ }^{3}$
}

1 Institut für Experimentelle Kernphysik, Karlsruher Institut für Technologie, 76128 Karlsruhe,

Germany, E-mail: anne.zillesakit.edu.

2 Dept. of Physics, University of Chicago, Chicago, IL 60637, USA.

3 Dept. of Physics and Astronomy, University of California, Los Angeles, CA 90095, USA.

4 Jet Propulsion Laboratory, Pasadena, CA 91109, USA.

5 Dept. of Physics, Grad. Inst. of Astrophys., \& Leung Center for Cosmology and Particle Astrophysics, National Taiwan University, Taipei, Taiwan.

6 Dept. of Physics, University of Delaware, Newark, DE 19716, USA.

7 Dept. of Physics and Astronomy, University of Hawaii, Manoa, HI 96822, USA.

8 SLAC National Accelerator Laboratory, Menlo Park, CA 94025, USA.

9 Institut für Kernphysik, Karlsruher Institut für Technologie, 76021 Karlsruhe, Germany.

10 Physics Dept., College of William \& Mary, Williamsburg, VA 23187, USA.

11 Dept. of Physics and Astronomy, University College London, London, United Kingdom.

12 Dept. of Physics, Washington University, St. Louis, MO 63130, USA.

13 Dept. of Physics, Stanford University, Stanford, CA 94305, USA.

\begin{abstract}
The SLAC T-510 experiment was designed to verify established microscopic models for simulation of radio emission from air-showers by reproducing the physics under controlled lab conditions. For this verification, the simulation toolkit Geant 4 was expanded by the calculation of the emitted radio signal with the "endpoint" and the "ZHS" formalisms in parallel. We present and compare the results of the two simulation approaches, taking into account the details of the experimental set-up such as the beam energy, target geometry and material, and the magnetic field configuration. We put special emphasis on the discussion of the effects due to refraction and transition radiation and show a comparison of the simulation results with the measured data of the SLAC T-510 Experiment.
\end{abstract}

The 34th International Cosmic Ray Conference,

30 July- 6 August, 2015

The Hague, The Netherlands

\footnotetext{
* Speaker.
} 


\section{Motivation}

The comparison of measured data from air-shower events caused by ultra-high energy cosmic rays with results from Monte-Carlo simulations is necessary to understand the characteristics of these air-showers. Two codes available for the simulation of the development of extensive air-showers are e.g. AIRES [1] and CORSIKA [2]. Both simulation codes split the continuous trajectories of the particles in to multiple sub-tracks. In addition to the fact that both simulation tools are based on different hadronic and electromagnetic interaction codes to handle particle interaction, they also use different models to calculate the radio emission by the simulated particle showers. The "endpoint" formalism [3][4] applied in CoREAS [5] based on CORSIKA and the "ZHS" formalism [6] applied in ZHAireS [7] based on AIRES. The two formalisms calculate the electric field of the moving charged particles in the shower. Both make no assumptions on the mechanism of radio emission, they only use the positions of the end-points of these sub-tracks and their corresponding times which are available by design as the basis for the calculation of radio emission from the simulated particle showers.

For the radio detection of the extensive air showers it is important to reduce the uncertainty in the models by validating them in a controlled laboratory environment. Therefore, the T-510 experiment at the SLAC National Accelerator Laboratory was designed to verify the radio emission mechanism from particle showers and can be used to compare the measured data to the predictions of Monte-Carlo simulations using both formalisms. An overview of the experimental setup of the T-510 experiment as well as the physics motivation are described in [8].

The SLAC electron beam delivers bunches of electrons with the primary energy of about $4.35 \mathrm{GeV}$ to $4.55 \mathrm{GeV}$ which are shot into a dielectric target made of high density polyethylene. In the target, the electrons induce a shower that mimics air-showers produced by high energy primary particles in the atmosphere. To study the variation of the radio signal produced as a function of magnetic field strength, the shower development takes place in a strong controllable magnetic field. The produced radio signal is recorded by antennas positioned on a vertical axis with respect to the beam axis. The measured horizontal polarisation component of the electric field can be interpreted as the contribution of the "geo"-magnetic effect [9], i.e. a linearly polarised electrical field proportional to the cross-product of the direction of the shower and the magnetic field vector. The vertical one can be interpreted as the contribution of the Askaryan effect [10], i.e. a linearly polarised electric field which is radially oriented around the shower axis.

In comparison to the results presented in [11] this contribution shows the results including the experimental set-up such as the beam energy, target geometry and material, as well as the realistic magnetic field configuration and effects due to refraction, transmission and transition radiation.

\section{Simulation scheme}

The simulation toolkit Geant4 10.0 is used. All details of the experimental setup, such as target geometry and material, as well as the beam particles and their energy were taken into account. All relevant interactions of shower photons and electrons are included in the simulation. To study the effect of a magnetic field on the emission of the radio signal, the measured map of the magnetic field strength in three space coordinates [12] is integrated in the simulation. Since the magnetic field strength scales linearly with the applied current, the strength of the field can be controlled by the current set during the measurements. The maximum applied current of $2400 \mathrm{~A}$ leads to a 
maximum magnetic field strength of about $970 \mathrm{G}$ along the vertical axis with respect to the beam. To minimize the effect of an artificial offset in the particle positions arising from the handling of multiple scattering in Geant4, we used a feature to set up a minimum sub-track size, so that no sub-track to exceed $0.2 \mathrm{~mm}$. This offset would otherwise lead to artificial velocities larger than the speed of light along the tracks and therefore to a possible overestimation of the emitted electric field. The simulations include the calculation of the radio signals produced by the particle showers in the target based on the sub-track positions and times given by Geant 4 . Each sub-track contributes to the calculations of the electric field or to the vector potential using the "endpoint" and "ZHS" formalisms which run in parallel. This provides a one-to-one comparison so that shower-to-shower fluctuations are not an issue in the comparison of the two formalisms. Depending on the number of electrons which was used in the simulation, the resulting electric field is then scaled up to the measured mean bunch charge of $131 \mathrm{pC}$ with a shot-to-shot standard deviation of $3.3 \mathrm{pC}$ [12]. To mimic the measured data, a 5-pol-butterworth filter is applied on the resulting signals for frequencies between $300-1200 \mathrm{MHz}$.

\subsection{Refraction and transmission effects}

Refraction at the upper slanted target boundary as well as Fresnel transmission coefficients and demagnification effects [13] are taken into account in the propagation of the radio signals. For every combination of track and antenna position, the point of refraction on the upper surface has to be found individually. To find the point of refraction, where the propagation time of the signal from the end-points of the sub-track to antenna is the shortest and its path fulfills the Snell's law, the coordinate system is transformed as shown in fig. 1 (left) where the target surface is defined as x-z-plane as shown in fig. 1 (left). The equation

$$
\left|Y_{t}\right| \cdot \tan \alpha=\left|Z_{d}\right|-\left|Y_{d}\right| \cdot \tan \left(\arcsin \left(\frac{n_{\mathrm{HDPE}}}{n_{\mathrm{Air}}} \cdot \sin \alpha\right)\right)
$$

can be derived from geometrical considerations, with $\left|Y_{t}\right|$ denoting the distance of the track to the surface and $\left|Y_{d}\right|$ the one for the antenna. The parameter $\left|Z_{d}\right|$ is the distance in $z$-direction to the point of refraction and $\alpha$ the angle of incidence. In the simulation all parameters are known and can be used to calculate the point of refraction numerically using equation 2.1 for every track and antenna combination, requiring that they intersect at the upper target surface. Once the point is known, it is possible to calculate the incident and emergent angle to the upper target surface and the corresponding Fresnel transmission coefficients for the vertical and horizontal polarisation components of the electric field with respect to the plane of incidence. This leads to a transmitted electric field of

$$
\vec{E}_{\text {ant }}=\left(\vec{E}_{\text {em }} \cdot \hat{\vec{r}}_{\perp, \text { in }}\right) \cdot t_{\perp} \cdot \hat{\vec{r}}_{\perp, \text { out }}+\left(\vec{E}_{\text {em }} \cdot \hat{\vec{r}}_{\|, \text {in }}\right) \cdot t_{\|} \cdot \hat{\vec{r}}_{\|, \text {out }} .
$$

The parameter $\vec{E}_{e m}$ is the electric field emitted by the track, $\hat{\vec{r}}_{\perp}$ the vector which is perpendicular to the plane of incidence (in) as well as after correction for refraction (out) and $\hat{\vec{r}}_{\|}$is the vector for the parallel case. The factors $t_{\perp}$ and $t_{\|}$are the corresponding Fresnel transmission coefficients [13] for the perpendicular and parallel polarisation component, respectively, of the electric field for each combination. Fig. 1 (right) the Fresnel transmission coefficients behavior as a function of the signal emergent angle. Larger emergent angles represent larger antenna heights with respect to the point of refraction, following a cosine-dependency. 
The time $t_{\text {prop }}$ for the signal propagation from track to antenna is obtained directly from using the point of refraction and is given by:

$$
t_{\text {prop }}=n_{\mathrm{HDPE}} \cdot \frac{d_{\mathrm{HDPE}}}{c}+n_{\mathrm{Air}} \cdot \frac{d_{\mathrm{Air}}}{c}
$$

where $d_{\mathrm{HDPE}}$ is the distance between track and point of refraction and $d_{\mathrm{Air}}$ the distance between point of refraction and antenna. The time $t_{\text {prop }}$ has then to be added to the time when the signal is emitted by the track.
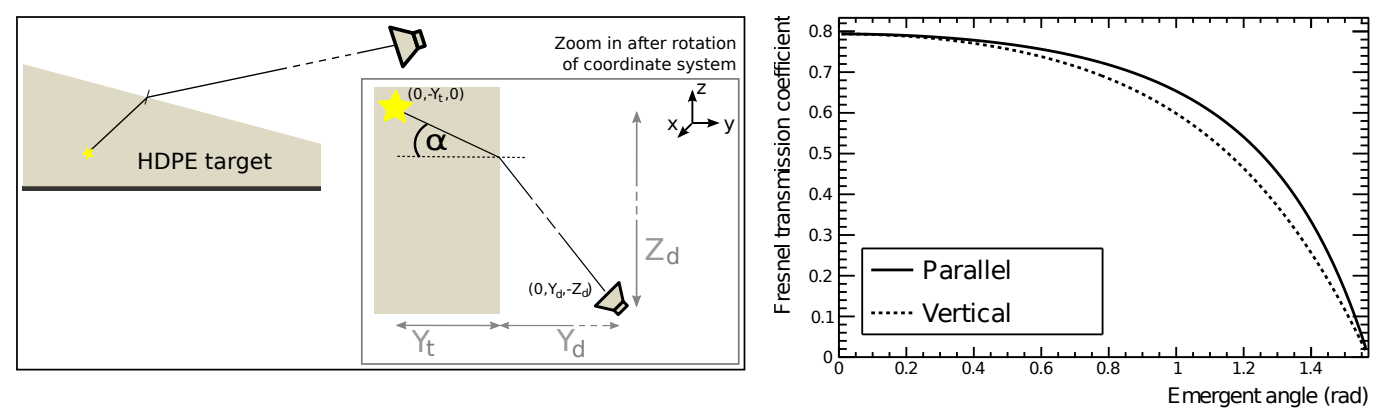

Figure 1: Left: sketch of experiment geometry for the calculation of the point of refraction at the upper slanted target surface; right: values of the Fresnel transmission coefficient for the electric field components parallel and perpendicular to the plane of incidence in dependency on the emergent angle.

It is difficult to properly account for reflections of radiation from the sides and bottom of the target as well as the reflections from structures inside the laboratory. To reduce reflections from the bottom, the target rests on absorptive material. The simulations do not currently include the reflections. Also the effect of diffraction is not implemented in the simulation. It is expected to be significant in the near-field zone only.

\section{Simulation results}

The SLAC T-510 experiment can manipulate the intensity of the signal strength in the horizontal polarisation component of the electric field signal by changing the strength of the magnetic field along the vertical axis from the beam axis [12]. Thus, it is possible to study the influence of the magnetic field on the emission of the radio signal. For the experiment it is expected that the measured electric field strength in the horizontal polarisation component rises linearly with rising magnetic field strength, due to the "geo"-magnetic effect, whereas the signal in the vertical polarisation component due to the Askaryan effect should be independent of the magnetic field.

The electric field in the time domain has been calculated using the "endpoint" formalism for an array of antenna locations, where the distance between the individual antennas is in $0.5 \mathrm{~m}$ steps in positions horizontal and vertical to the beam axis with a primary electron energy of $4.35 \mathrm{GeV}$. The distance to the entrance point of the electrons in the target is about $13 \mathrm{~m}$. The results for the maximum peak amplitudes of the electric field for an applied magnetic field is shown in figure 2 (left). To arrive at this result, in Geant4 simulation, a magnetic field using the values from the measured magnetic field map is switched on which has the maximum strength of up to $970 \mathrm{G}$ in the vertical 


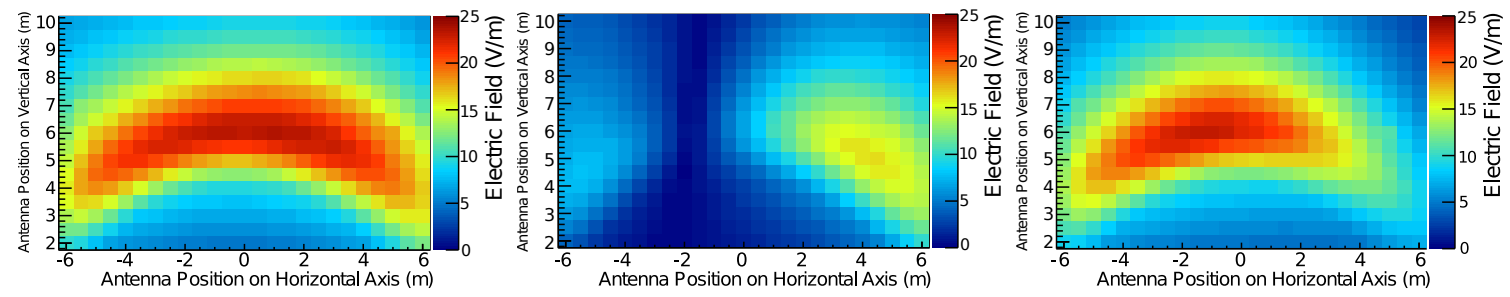

Figure 2: Peak amplitude of the electric field for a 2D antenna array using the "endpoint" formalism for a magnetic field of a maximum strength $970 \mathrm{G}$ : left: total electric field; center: horizontally polarised component; right: vertically polarised component.
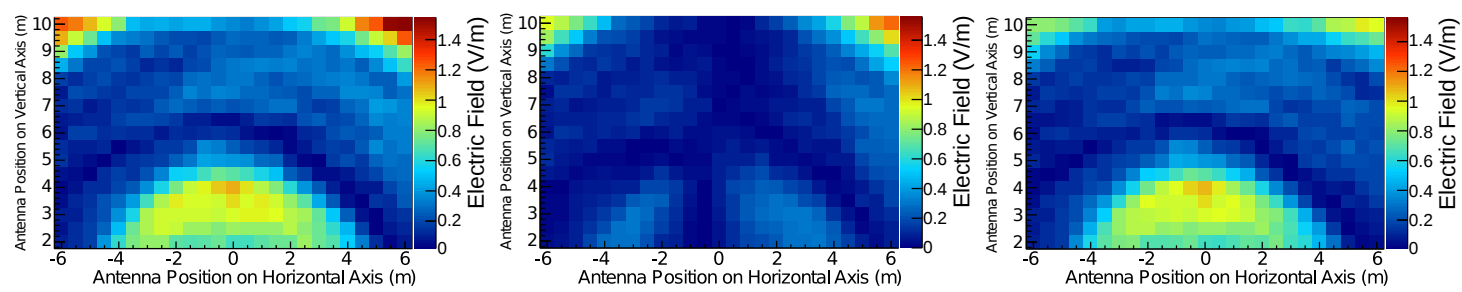

Figure 3: Contribution of transition radiation using "endpoint" formalism for a magnetic field of 970 Gauss; peak amplitude in time domain for: left: total electric field; center: horizontal polarisation component; right: vertical polarisation component.

direction perpendicular to the electron beam. Figure 2 shows also the results for the horizontal (center) and vertical (right) polarisation components. The positions of the maximum values for the peak amplitude of the signal form a strong Cherenkov ring, whose position agrees with the expectation given by the refractive index of $n_{\mathrm{HDPE}}=1.52$ and $n_{\text {Air }}=1.0003$. The finite target size leads to a cut-off of the Cherenkov ring on both sides. The more elliptical than circular appearance of the ring is caused by the refraction at the slanted target surface. Switching on the magnetic field leads to an asymmetric rise of the total signal amplitude on the ring structure to one side. The impact of the "geo"-magnetic effect is observable especially in the horizontal polarisation component of the electric field (see fig. 2, center). A comparison to the results with no applied magnetic field can be found in [11]. The results for the "ZHS" formalism are not shown, as they are nearly identical to those for the "endpoint" formalism (see fig. 5).

\subsection{Impact of transition radiation}

The passage of the beam out of the boundary of the lead preshower into the target (where the main part of the shower develops) is expected to produce transition radiation. Its influence on the measured signal can be studied using the "endpoint" formalism. Because radiation can not escape out of metal, just the start of the first sub-track of particle trajectory which passes the boundary has to be taken into account. The results for a 2D antenna array are shown in fig. 3. A small asymmetry is visible because the magnetic field starts to affect the shower during the preshower stage. For antennas close to the Cherenkov angle, the contribution of transition radiation is about $1 \%$ for the horizontal and vertical polarisation components (compare the scale to fig. 2, top) and is therefore negligible for the these studies. 


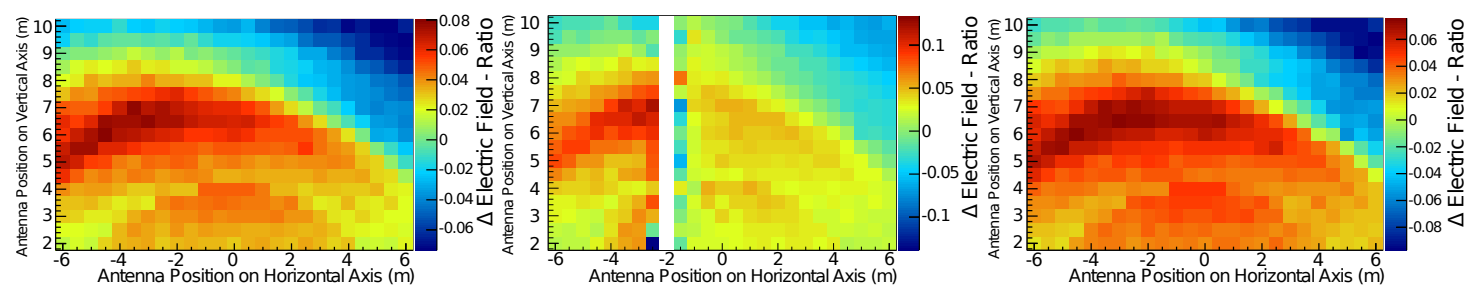

Figure 4: Ratio in peak amplitude of total electric field in time domain for a 2D antenna array defined as $\frac{E_{\mathrm{ZHS}}-E_{\text {endpoint }}}{E_{\mathrm{ZHS}}}$ : left: difference in total electric field; center: difference in horizontally polarised component, where antenna positions with negligible signals have been excluded; right: difference in vertically polarised component.

\subsection{Comparison of the "endpoint" and "ZHS" formalisms}

The 2D distribution in figure 4 (left) shows the relative deviation of the peak amplitude in the time domain for the "endpoint" formalism to the one for the "ZHS" formalism for the maximum magnetic field strength of $970 \mathrm{G}$ using the realistic field map and a primary beam energy of $4.35 \mathrm{GeV}$. The deviations corresponds to about $7-8 \%$ for the total peak amplitude and depend on the position of the antenna. The deviations on the horizontal (center) and vertical component (right) show that the "endpoint" and "ZHS" formalism lead to the best agreement in the area of the highest peak amplitude values if a magnetic field is applied. This leads to the assumption that the formalisms reproduce the contribution due to the "geo"-magnetic effect in a slightly different way. Furthermore, it is visible that inside the Cherenkov ring the "ZHS" formalism leads to slightly higher results and the "endpoint" formalism pedicts slightly higher amplitudes outside the ring.

For a detailed comparison, figure 5 (top) shows the vertical and horizontal polarisation components of the electric field in the time domain for both formalisms for an antenna position close to the Cherenkov angle at $6 \mathrm{~m}$ height on the vertical axis with respect to the beam. The shapes of the frequency spectra agree very well (see fig. 5, bottom left), except for a deviation of about 3-7\% (see fig. 5, bottom right) between $300-1200 \mathrm{MHz}$. The direct comparison of the peak amplitude in the time domain gives an agreement for both models for the horizontal polarisation component within $4.4 \%$ and for the vertical polarisation component within $6.4 \%$. In the time and frequency domains, the signal shows a large discrepancy in the in the vertical polarisation component than in the horizontal one. This deviation can be interpreted as difference between the two formalisms, because the "ZHS" and the "endpoint" formalisms run in parallel and are using the same routines for calculation of refraction and transmission at the surface. In future work the origin of this difference should be investigated.

\section{Comparison to data}

An overview of the measurements and calibration are described in [12]. The simulations for comparison with data are done by injecting a bunch of 5000 electron primaries, to reduce statistical fluctuations, with an energy of $4.35 \mathrm{GeV}$ each. The simulated signals for both formalisms are convolved with the effective height and measured system response of the antennas to compare with the data [12], so that they can be compared with the measured voltages of the data directly. The filtering was done using the hardware filters and downsampling the simulated data. Figure 6 shows the horizontal polarisation component of the electric field for an antenna position close to the 

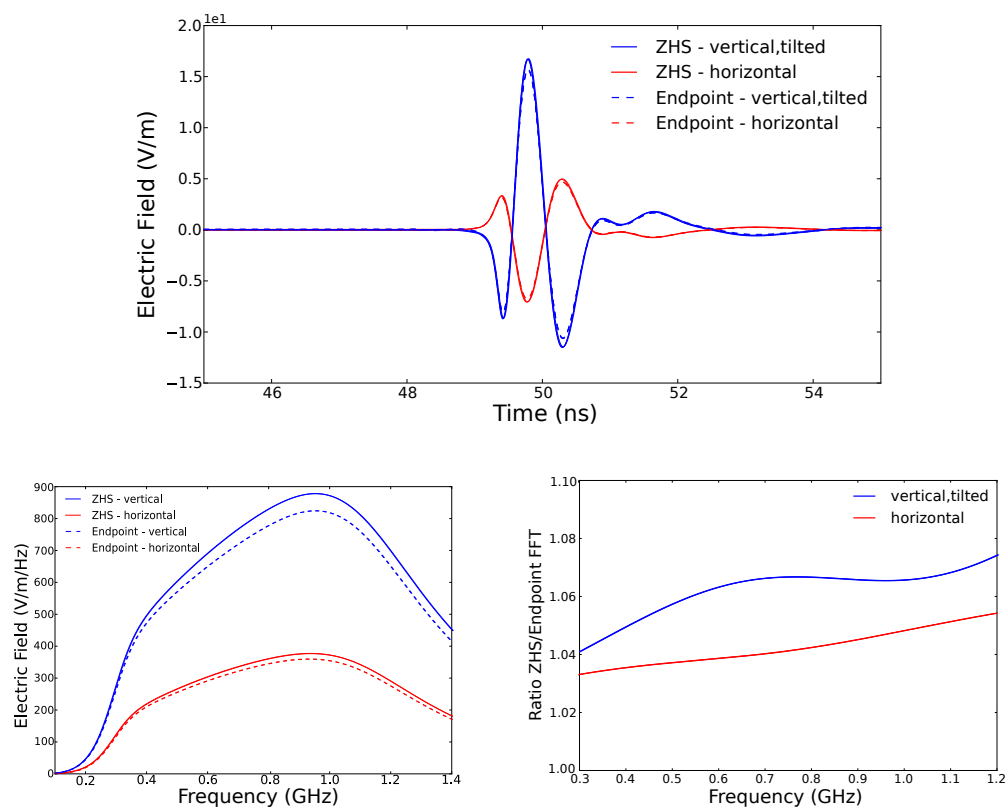

Figure 5: Comparison of "ZHS" and the "endpoint" formalisms using an antenna at the vertical axis at a height of $6 \mathrm{~m}$ with a primary energy of $4.35 \mathrm{GeV}$ and a magnetic field strength of about 970 G: top: signals in time domain; bottom left: signals in frequency domain; bottom right: ratio of frequency spectra from "ZHS" and "endpoint" simulations.

Cherenkov angle at $6.52 \mathrm{~m}$ height above the beam in a distance of about $13.5 \mathrm{~m}$ from the entrance point of the electrons in the target. The maximum peak amplitudes in time domain for the two formalisms agree within 3\% for this antenna position. The agreement of the shape in the time domain for data and simulation is clearly visible. The radio emission of the first order internal reflection from the bottom of the target is expected to arrive at the antennas $\sim 1 \mathrm{~ns}$ after the main pulse. In the measurements, this reflection interferes with the main peak constructively. The calculation of the percent difference between the simulated signal without and with adding the total reflected signal from the bottom of the target at the expected time leads to the conservative assumption of a systematic uncertainty of $38 \%$. Finally, a comparison of the maximum peak amplitude in the time domain shows that the simulated data using the two formalisms agree with the measured data within about $35 \%[12]$.

\section{Conclusion}

There are two established formalisms for the simulation of radio emission by extensive particle showers. To validate these formalisms, the SLAC T-510 experiment was performed to approximate air-shower conditions in a controlled laboratory environment where the impact of the "geo"-magnetic effect could be examined. Geant4 simulations including realistic beam energy, target geometry and material, and the magnetic field configuration were done using the "endpoint" and "ZHS" formalisms in parallel. It has been shown that the impact of the transition radiation is negligible in this experiment. Results for both formalisms were shown and are found to be consistent within about $3-7 \%$, and the Cherenkov ring structure is clearly visible in both electric field polarisation components. The comparison of the simulation predictions to the measured 


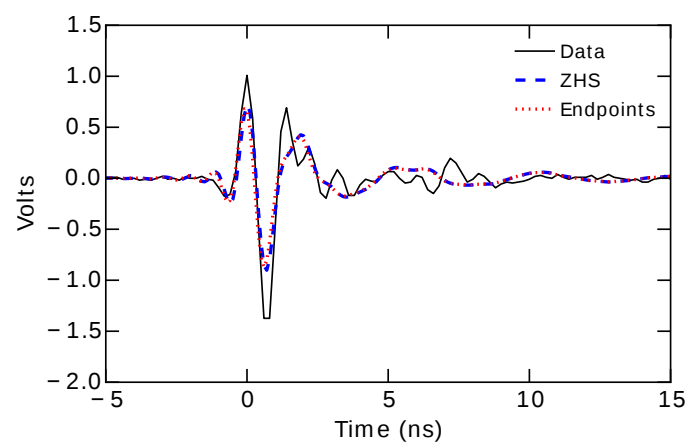

Figure 6: Comparison of the measured signal in the time domain with the resulting radio signal in the horizontal component for "ZHS" and the "endpoint" formalisms using an antenna at the vertical axis at a height of $6.52 \mathrm{~m}$ with a primary energy of $4.35 \mathrm{GeV}$ and a magnetic field strength of about $970 \mathrm{G}$.

data of the SLAC T-510 experiment shows an agreement for both formalisms within 35\% which is well consistent within the systematic uncertainty of $38 \%$. Internal reflections within the target are the dominant contributor to the systematic uncertainty in the comparison between measured and Monte-Carlo data.

Acknowledgments. The authors thank SLAC National Accelerator Center for providing facilities and support and especially Janice Nelson and Carl Hudspeth for their support and dedication that made T-510 possible. Work supported in part by Department of Energy contract DE-AC0276SF00515 and by the "Helmholtz Alliance for Astroparticle Phyics HAP" funded by the Initiative and Networking Fund of the Helmholtz Association.

\section{References}

[1] S. J. Sciutto, arXiv:astro-ph/9911331.

[2] D. Heck, The Air Shower Simulation Program CORSIKA, https://web.ikp.kit.edu/corsika/.

[3] C. W. James, H. Falcke, T. Huege and M. Ludwig, arXiv:1007.4146 [physics.class-ph].

[4] M. Ludwig, T. Huege, Astroparticle Physics 34, 438-446, 2011.

[5] T. Huege, M. Ludwig, C. W. James, arXiv:1301.2132 [astro-ph.HE].

[6] J. Alvarez-Muniz, A. Romero-Wolf, E. Zas, arXiv:1002.3873v1 [astro-ph.HE].

[7] J. Alvarez-Muniz, W. R. Carvalho Jr., E. Zas, arXiv:1107.1189 [astro-ph.HE].

[8] K. Belov et al., SLAC T-510: Geant4 simulations of radio signals from particle showers for the SLAC T-510 experiment, see contribution 346 of this conference.

[9] F. D. Kahn, I. Lerche,Proc. of Royal Society of London, Series A, Mathematical and Physical Sciences, 289:206, 1966; DOI: 10.1098/rspa.1966.0007.

[10] G. A. Askaryan, Sov. Phys. JETP 14, 441, 1965.

[11] A. Zilles et al., Geant4 simulations of radio signals from particle showers for the SLAC T-510 experiment, ARENA proceedings 2014.

[12] S. Wissel et al., Measurements, system response, and calibration of the SLAC T-510 Experiment, see contribution 342 of this conference.

[13] N. G. Lehtinien, P. W. Gorham, A. R. Jacobson, R. A. Roussel-Duprée, FORTE satellite constraints on ultrahigh energy cosmic particle fluxes, Phys. Rev. D 69 013008, 2004. 